\title{
Obtenção da veia safena magna através de acesso minimamente invasivo para revascularizações miocárdicas
}

\author{
André Luiz TYSZKA*, Leila Satomi FUCUDA*, Eloisa de Brida TORMENA**, \\ Antonio Carlos L. CAMPOS***
}

RBCCV 44205-536

Tyszka A L, Fucuda L S, Tormena E B, Campos A C L - Obtenção da veia safena magna através de acesso minimamente invasivo para revascularizações miocárdicas. Rev Bras Cir Cardiovasc 2001; 16(2): 105-113.

RESUMO: Objetivo: Avaliar a possibilidade de obtenção da veia safena magna através de miniincisões de pele, a sua qualidade e a ocorrência de complicações precoces da ferida operatória.

Casuística e Métodos: Foram estudados 46 pacientes, admitidos entre julho e novembro de 1999. Após miniincisões longitudinais de pele a veia safena magna foi identificada e, com auxílio de afastador de lâmina longa e estreita, delicadamente dissecada. Os pacientes foram divididos em 2 grupos na dependência da presença dos fatores de risco: anemia, aterosclerose periférica, obesidade e diabete melito. As feridas operatórias foram observadas quanto às complicações maiores e menores. Amostras da veia foram enviadas para estudo histológico.

Resultados: O número médio de incisões foi 2,3, com tamanho médio de $3,5 \mathrm{~cm}$ e de todas incisões somadas de $7,3 \mathrm{~cm}$. O tamanho médio da veia foi $34,1 \mathrm{~cm}$, com tempo médio de retirada de 28,7 minutos. Foram observadas complicações menores em $5(10,8 \%)$ pacientes; sendo hematoma local a mais comum (6,5\%). Não foram observadas complicações maiores e a ressecção foi sempre possível. Em 2 casos houve lesão macroscópica da veia, sendo possível a sua correção e utilização. O estudo histológico demonstrou preservação da arquitetura tecidual e não evidenciou lesão endotelial significativa.

Conclusões: A obtenção da veia safena magna através de miniincisões é possível e resulta em adequado enxerto venoso. A incidência de complicações da ferida é baixa e independente dos fatores de risco. Estes resultados preliminares sugerem que a técnica pode ser aplicada com segurança em pacientes submetidos à revascularização miocárdica, embora os resultados a longo prazo ainda necessitem ser determinados.

DESCRITORES: Revascularização miocárdica. Procedimentos cirúrgicos cardíacos, métodos. Veia safena, cirurgia, métodos. Procedimentos cirúrgicos minimamente invasivos. Veia safena, transplante.

Trabalho realizado no Instituto do Coração de Maringá - Hospital Santa Rita de Maringá. Maringá, PR, Brasil.

Recebido para publicação em maio de 2000.

* Do Serviço de Cirurgia Cardiovascular do Hospital Santa Rita de Maringá.

** Do Fênix Laboratório da Patologia.

*** Do Departamento de Cirurgia do Hospital de Clínicas da Universidade Federal do Paraná.

Endereço para correspondência: André Luiz Tyszka. Av. XV de Novembro, 331, apto. 304. Centro. Maringá, PR, Brasil. CEP: 87013-230. Tel.: (44)

222-5864. Fax (44) 225-7333. e-mail: altyszka@uol.com.br 
Tyszka A L, Fucuda L S, Tormena E B, Campos A C L - Obtenção da veia safena magna através de acesso minimamente invasivo para revascularizações miocárdicas. Rev Bras Cir Cardiovasc 2001; 16(2): 105-13.

\section{INTRODUÇÃO}

O tratamento cirúrgico da isquemia miocárdica completou três décadas desde os primeiros relatos da revascularização coronariana direta. Neste período se tornou um dos procedimentos isolados mais amplamente estudados e realizados na história da cirurgia (1). Desde a publicação por LOOP et al. (2), em 1986, a artéria torácica interna esquerda é o enxerto de escolha para as anastomoses na artéria interventricular anterior. Em 1999, LYTLE et al. (3) documentaram a superioridade da utilização das duas artérias torácicas internas. A utilização clínica dos outros enxertos arteriais, como a artéria radial e a artéria gastroepiplóica, é bem estabelecidada, mas seus resultados tardios ainda estão por serem determinados. Mesmo com a maior aplicação dos enxertos arteriais, a veia safena magna ainda é o enxerto mais freqüentemente utilizado para as revascularizações miocárdicas (4). Para a sua dissecção, a técnica tradicional e comumente utilizada é através de longa e única incisão acompanhando o trajeto venoso. As complicações deste tipo de incisão no membro inferior são bem conhecidas. Complicações maiores que resultam em reoperação ou em prolongamento do internamento hospitalar não são freqüentes (1-6\%) ${ }^{(5,6)}$. Contudo, as complicações menores, que levam à dificuldade de cicatrização, podem atingir até $44 \%$ dos pacientes ${ }^{(7-}$ 9). A infecção no membro inferior é cerca de 99 vezes mais freqüente do que na incisão esternal $e$, na grande maioria das vezes, representa as principais queixas dos pacientes no pós-operatório (10).

Resultados iniciais demonstraram que os procedimentos menos invasivos mantém a efetividade do tratamento cirúrgico, aceleram a recuperação dos pacientes, diminuem o tempo de permanência hospitalar e reduzem os custos globais ${ }^{(11)}$. Com este direcionamento, a dissecção minimamente invasiva da veia safena magna despertou a atenção dos pesquisadores. Estudos preliminares, com o uso dos videoendoscópicos, demonstraram que as incisões operatórias limitadas são capazes de reduzir a morbidade pós-operatória (12). Porém, as operações videoendoscópicas necessitam da aquisição de novas habilidades e novos equipamentos. O período inicial de curva de aprendizado pode comprometer os resultados e a necessidade de investimentos para aquisição deste material podem ser proibitivos em inúmeras Instituições (13).

Visando atingir os benefícios dos acessos minimamente invasivos, sem a utilização da videoendoscopia, métodos alternativos de obtenção da veia safena magna foram estudados. Através de miniincisões e com o auxílio de um afastador de lâmina longa e estreita, pode ser dissecado o segmento necessário de veia ${ }^{(14)}$. Em 1997, DIAS et al. (15) apresentaram uma série inicial de 8 pacientes nos quais técnica semelhante foi empregada. Através de miniincisões de pele puderam ressecar comprimento satisfatório de veia, com baixos índices de complicações da ferida. Contudo, não foi avaliado se essa forma de obtenção da veia safena magna pode comprometê-la morfologicamente.

O presente estudo teve como objetivos: a) avaliar a possibilidade de obtenção de comprimento necessário de veia safena magna através de miniincisões de pele para as revascularizações miocárdicas; b) avaliar a evolução precoce da ferida operatória do membro inferior; e c) avaliar a qualidade do enxerto, mediante análise macroscópica e histológica.

\section{CASUÍSTICA E MÉTODOS}

Foram estudados 47 pacientes submetidos consecutivamente à revascularização miocárdica, 29 do sexo masculino e 18 do sexo feminino, com idade variando de 42 a 82 anos $(61,5 \pm 9,9)$, admitidos no período de julho a novembro de 1999, pelo Instituto do Coração de Maringá.

Após a tricotomia da região operatória, os pacientes eram encaminhados ao centro cirúrgico. Recebiam, por via intravenosa, 1,0 g de cefazolina sódica e com o paciente anestesiado era realizada a limpeza da região operatória com solução de Povidine Degermante ${ }^{\circledR}$ e a antissepsia com solução de Povidine Tintura ${ }^{\circledR}$.

O membro inferior era posicionado com abdução parcial da coxa, rotação externa e leve flexão do quadril, obtida com o auxílio de dois campos cirúrgicos colocados inferiormente à coxa e ao joelho do paciente, conforme ilustrado na Figura 1.

\section{Técnica Operatória}

\section{Dissecção da veia safena magna:}

A primeira incisão, com cerca de $3 \mathrm{~cm}$, era realizada no sentido longitudinal, no trajeto da veia safena magna, $10 \mathrm{~cm}$ abaixo da prega inguinal (Figura 2). O tecido celular subcutâneo e o tecido adiposo eram dissecados e a veia identificada com o auxílio de afastador de lâmina longa e estreita (Sauerbruch Retractor, - Boss Instruments Ltda. ${ }^{\circledR}$ ) - Figura 3.

Procedia-se à dissecção cuidadosa da veia em sua extensão, primeiramente em direção cefálica, até à junção safeno-femoral. $O$ plano anterior da veia safena magna, que geralmente não apresenta 
Tyszka A L, Fucuda L S, Tormena E B, Campos A C L - Obtenção da veia safena magna através de acesso minimamente invasivo para revascularizações miocárdicas. Rev Bras Cir Cardiovasc 2001; 16(2): 105-13.

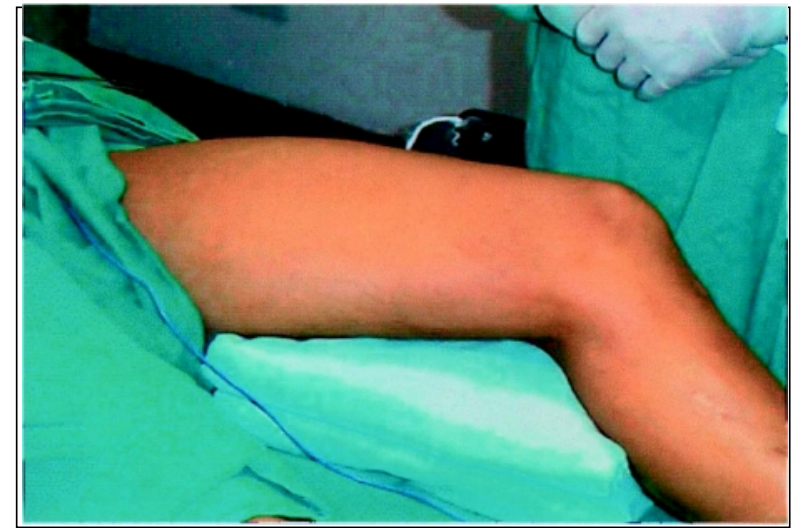

Fig. 1 - Posicionamento do membro inferior esquerdo (paciente 39).

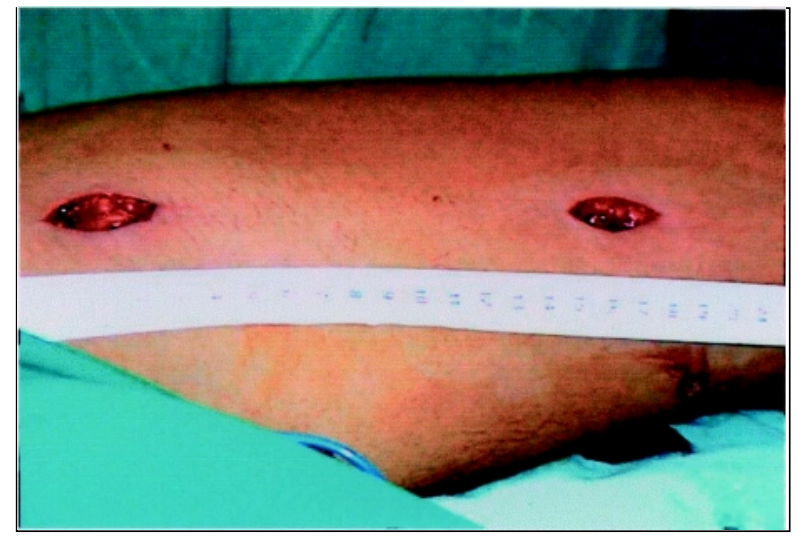

Fig. 2 - Miniincisões sobre o trajeto da veia safena magna esquerda (paciente 39).

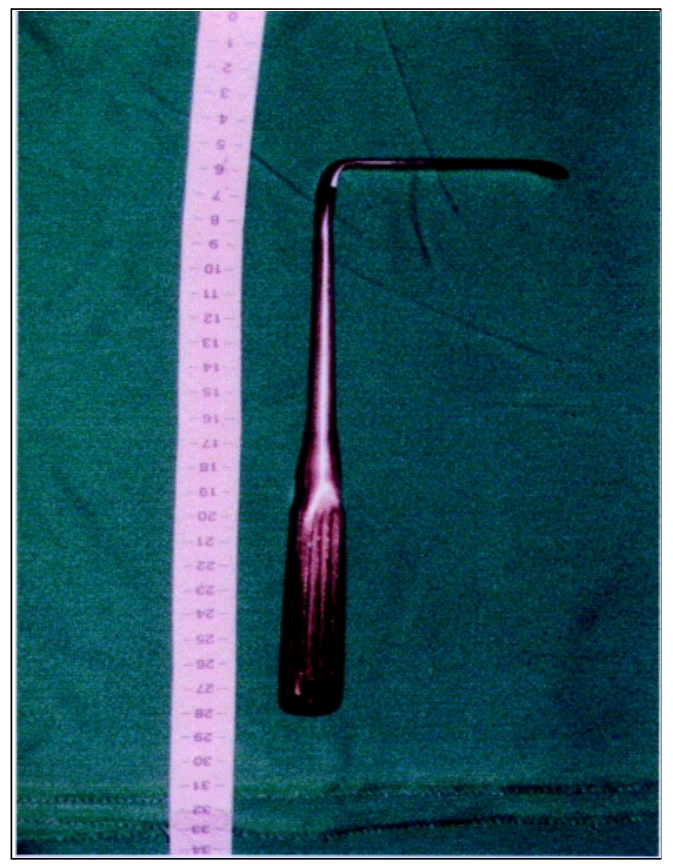

Fig. 3 - Afastador Sauerbruch (BOSS Instruments Ltda®). colaterais, era dissecado com tesoura de Metzenbaum e manobras digitais. A lâmina do afastador era, então, introduzida neste plano, paralelamente ao comprimento da veia. A dissecção lateral e inferior era realizada sob visão direta. Os ramos colaterais eram identificados e ligados na sua porção distal com clipe metálico e, a seguir, seccionados. A porção proximal dos ramos colaterais, junto à veia, apresenta vasoespasmo imediatamente após o corte e, na maioria das vezes, não há sangramento. A ligadura dos ramos era realizada posteriormente durante a preparação da veia ex situ.

Seguindo desta maneira cerca de 6 a $10 \mathrm{~cm}$ de veia puderam ser preparados de cada lado da incisão, o que permitiu distanciar 12 a $15 \mathrm{~cm}$ entre cada incisão seqüencial (Figura 2). O número de incisões era determinado pelo comprimento necessário de veia. As duas extremidades eram ligadas com fio de algodão trançado número 2-0, e a veia era retirada do seu leito (Figura 4).

O fechamento da ferida operatória era realizado em dois planos com fio polifilamentar absorvível $\left(\right.$ Vicryl $^{\circledR}$ 2-0 e Vicryl ${ }^{\circledR}$ 3-0 - Ethicon $\left.{ }^{\circledR}\right)$. Era colocado dreno laminar número 2 no leito da veia (Figura 5). O membro inferior, ao final da operação, era enfaixado com atadura de crepe de $20 \mathrm{~cm}$.

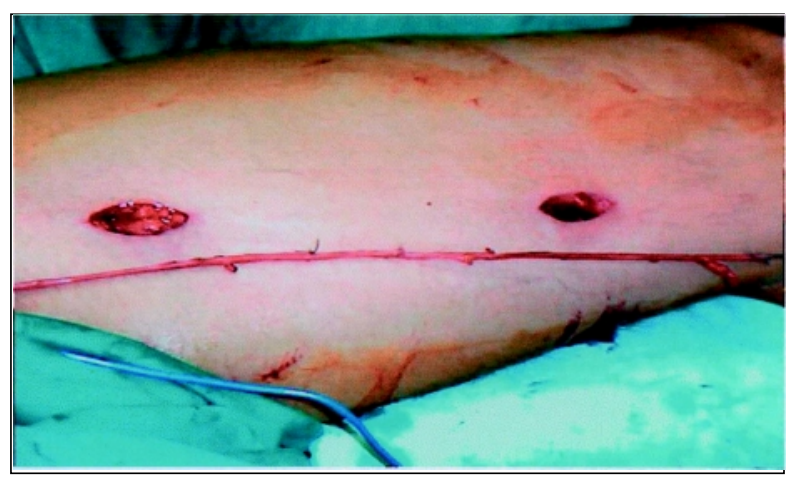

Fig. 4 - Segmento da veia safena magna após a retirada (paciente 39).

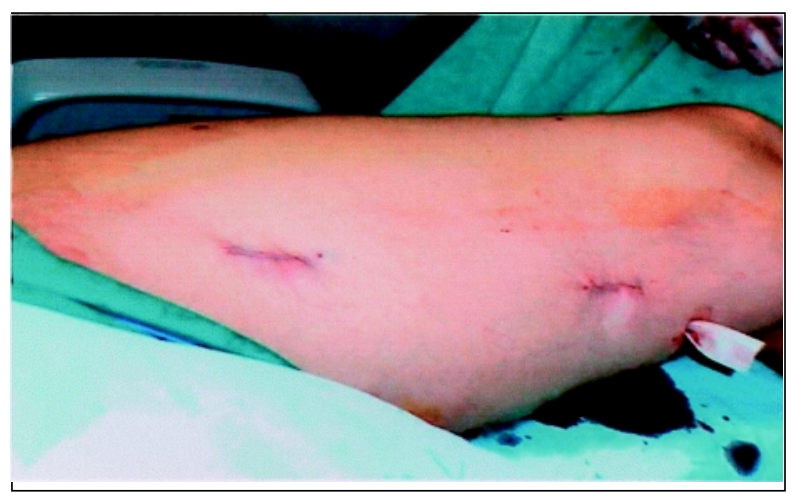

Fig. 5 - Aspecto final após a sutura da pele (paciente 39). 
Tyszka A L, Fucuda L S, Tormena E B, Campos A C L - Obtenção da veia safena magna através de acesso minimamente invasivo para revascularizações miocárdicas. Rev Bras Cir Cardiovasc 2001; 16(2): 105-13.

Os curativos eram trocados diariamente e o dreno laminar retirado no primeiro dia. A ferida operatória no membro inferior era avaliada diariamente até o quinto dia de pós-operatório. Após a alta, o paciente retornava para reavaliação ambulatorial em 15 dias de pós-operatório.

Os seguintes aspectos pré-operatórios foram analisados: idade, sexo e presença de fatores de risco preditivos para problemas cicatriciais do membro inferior - diabete melito, aterosclerose periférica, obesidade, anemia (hematócrito inferior a 35\%) (7,16-19). Os pacientes foram divididos em 2 grupos: Grupo 1, denominado grupo sem risco, correspondente aos pacientes que não apresentavam fatores de risco e Grupo 2, denominado grupo risco, correspondente aos pacientes com um ou mais fatores de risco definidos acima.

Os seguintes aspectos operatórios foram analisados: o procedimento cirúrgico (número de coronárias revascularizadas e procedimentos associados), as incisões (número, local, comprimento individual, comprimento total das incisões somadas) e a veia (comprimento, tempo para a retirada e lesões macroscópicas).

Os seguintes aspectos pós-operatórios da ferida foram avaliados: presença de hematoma, infecção local, reação inflamatória local, deiscência de sutura, necrose das bordas da incisão, dor e outras complicações. A dor foi classificada em intensa, quando necessitava de analgésicos parenterais; moderada: quando era aliviada com analgésicos via oral; leve quando estava presente durante o esforço físico e era aliviada com repouso, não necessitando de medicações e ausente ${ }^{(20)}$.

\section{Avaliação da Qualidade da Veia}

\section{Avaliação Macroscópica}

Imediatamente após a ressecção da veia de seu leito, amostras de aproximadamente $1 \mathrm{~cm}$ eram retiradas de cada extremidade e acondicionadas em solução de formaldeído a $10 \%$ para posterior análise histológica. Durante a preparação, a parede da veia era visualmente avaliada quanto à presença macroscópica de lesões decorrentes da dissecção e considerada adequada ou não.

\section{Avaliação Histológica}

Os cortes histológicos foram corados com hematoxilina-eosina ( $\mathrm{HE})$, para análise da arquitetura histológica e do endotélio e com a coloração de Calleja sem permanganato de potássio para a avaliação do tecido conjuntivo e das fibras elásticas (21). Para a análise da presença de traumas à parede venosa foram avaliadas a superfície endotelial e a arquitetura do tecido através das interações entre o tecido conjuntivo, as fibras elásticas e as fibras musculares lisas. A preservação do endotélio foi graduada de 1 a 6 , com o grau 1 representando a ótima preservação endotelial e grau 6 indicando máximo dano endotelial (22). As fibras elásticas foram avaliadas quanto à rotura e ao desarranjamento das fibras e, de acordo com estes achados, a arquitetura histológica foi classificada em normal ou alterada (23).

\section{Análise Estatística}

As variáveis quantitativas foram representadas através de seu valor mínimo e máximo, média e desvio padrão $(x \pm D P)$. Para a análise do dados nominais utilizou-se o teste exato de Fisher. O nível de significância estabelecido para a análise foi de 0,05 ou $5 \%$.

\section{RESULTADOS}

Quarenta e seis $(97,9 \%)$ pacientes completaram os procedimentos metodológicos propostos. Houve um óbito no período de pós-operatório imediato por síndrome de baixo débito.

\section{Dados Pré-Operatórios}

Dos 46 pacientes submetidos à operação, 21 $(45,6 \%)$ apresentavam um ou mais fatores de risco para complicações cicatriciais do membro inferior (Tabela 1), constituindo o Grupo 2.

\section{Dados Operatórios}

Quarenta e dois pacientes foram submetidos a revascularizações miocárdicas isoladas e $4(8,7 \%)$ pacientes sofreram procedimentos cirúrgicos associados (Tabela 2). A quantidade de vasos coronarianos revascularizados variou de 1 a $4(2,9$ $\pm 0,8)$. A artéria torácica interna esquerda foi utilizada em 43 (93,5\%) pacientes.

O número de incisões realizadas para obtenção do comprimento necessário de veia variou de 1 a 4 $(2,3 \pm 0,71)$. O tamanho de cada incisão variou de $2,5 \mathrm{~cm}$ a $6 \mathrm{~cm}(3,5 \pm 0,66)$. O comprimento total das incisões somadas em cada paciente variou de 3,5 $\mathrm{cm}$ a $13 \mathrm{~cm}(7,3 \pm 2,1)$. O comprimento da veia ressecada variou de $18 \mathrm{~cm}$ a $54 \mathrm{~cm}(34,1 \pm 7,8)$ Tabela 3. O Gráfico 1 demonstra a distribuição do número de casos agrupados em categorias de tamanho das incisões. Pode-se observar que $63 \%$ dos pacientes tiveram os comprimentos das incisões somadas inferiores ou iguais a $7,5 \mathrm{~cm}$. 
Tyszka A L, Fucuda L S, Tormena E B, Campos A C L - Obtenção da veia safena magna através de acesso minimamente invasivo para revascularizações miocárdicas. Rev Bras Cir Cardiovasc 2001; 16(2): 105-13.

TABELA 1

\begin{tabular}{lcc}
\hline \multicolumn{2}{c}{$\begin{array}{c}\text { NÚMERO DE PACIENTES COM FATORES DE RISCO } \\
\text { PARA COMPLICAÇÕES NA FERIDA DO MEMBRO } \\
\text { INFERIOR }\end{array}$} \\
\hline FATORES DE RISCO & $\mathbf{n}$ & $\%$ \\
\hline Diabete melito & 14 & $30,4 \%$ \\
Aterosclerose periférica & 7 & $15,2 \%$ \\
Anemia & 2 & $4,3 \%$ \\
Obesidade & 8 & $17,4 \%$ \\
Total & 21 & $45,6 \%$ \\
\hline $\mathrm{n}=$ número de pacientes & & \\
\hline
\end{tabular}

TABELA 2

PROCEDIMENTOS REALIZADOS

\begin{tabular}{lrr}
\hline PROCEDIMENTO & $\mathbf{n}$ & $\%$ \\
\hline RVM isoladas & 42 & $91,3 \%$ \\
RVM + TVAo & 2 & $4,3 \%$ \\
RVM + TVMi & 2 & $4,3 \%$ \\
\hline
\end{tabular}

$\mathrm{n}=$ número de pacientes; $\mathrm{RVM}=$ revascularização miocárdica; TVAo $=$ Troca de valva aórtica; TVMi = Troca de valva mitral.

TABELA 3

\begin{tabular}{|c|c|c|c|}
\hline \multicolumn{4}{|c|}{$\begin{array}{l}\text { MEDIDAS DAS INCISÕES (NÚMERO, TAMANHO E } \\
\text { COMPRIMENTO TOTAL) E DA VEIA OBTIDA }\end{array}$} \\
\hline & VALOR MIN & VALOR MAX & MÉDIA — DP \\
\hline Número & 1 & 4 & $2,3 \pm 0,7$ \\
\hline Tamanho & $2,5 \mathrm{~cm}$ & $6 \mathrm{~cm}$ & $3,5 \pm 0,7$ \\
\hline Comp. Total & $3,5 \mathrm{~cm}$ & $13 \mathrm{~cm}$ & $7,3 \pm 2,1$ \\
\hline Comp. da veia & $18 \mathrm{~cm}$ & $54 \mathrm{~cm}$ & $34,1 \pm 7,8$ \\
\hline
\end{tabular}

O tempo para retirada da veia variou de 12 a 45 minutos $(28,7 \pm 8,0)$. Considerando que o comprimento médio de veia ressecada foi de $34,1 \mathrm{~cm}$, obteve-se tempo médio de 0,8 minutos para cada centímetro de veia ressecada. Conforme observado no Gráfico 2, o histograma demonstra que a maioria $(65,2 \%)$ dos segmentos venosos foram obtidos com intervalo de tempo igual ou inferior a 30 minutos.

A ressecção da veia safena magna foi possível em todos os casos, não havendo necessi-
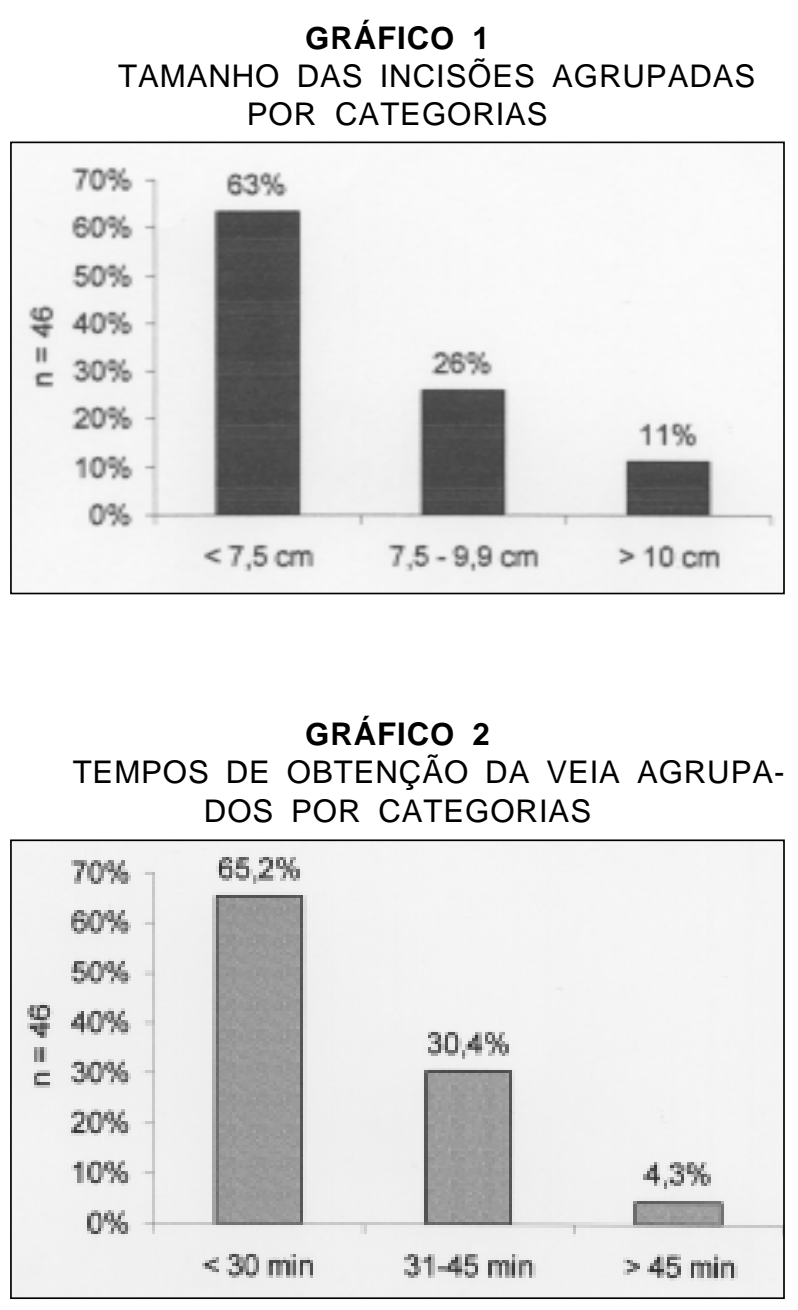

dade de conversão para a técnica convencional em nenhum paciente.

\section{Dados Pós-Operatórios}

Ocorreram complicações menores em 5 (10,8\%) pacientes e estão descritas na Tabela 4.

Dos 21 pacientes do Grupo 2 ou grupo risco, 2 (9,5\%) apresentaram complicações menores e dos 25 pacientes do Grupo 1 ou grupo sem risco, 3 (12\%) apresentaram complicações. A diferença entre os 2 grupos não se mostrou estatisticamente significativa $(p=1,0)$. Com relação ao sexo, dos 17 pacientes do sexo feminino, $2(11,8 \%)$ apresentaram complicações e dos 29 do sexo masculino 3 $(10,3 \%)$ tiveram complicações menores $(p=0,99)$.

Não foram observadas complicações como infecção local, deiscência total da sutura, necrose completa das bordas, hematoma extenso ou qualquer complicação maior que determinasse a 
Tyszka A L, Fucuda L S, Tormena E B, Campos A C L - Obtenção da veia safena magna através de acesso minimamente invasivo para revascularizações miocárdicas. Rev Bras Cir Cardiovasc 2001; 16(2): 105-13.

TABELA 4

COMPLICAÇÕES DA FERIDA OPERATÓRIA DE ACORDO COM O MEMBRO INFERIOR OPERADO E COM A PRESENÇA DE FATORES DE RISCO PARA COMPLICAÇÕES LOCAIS

\begin{tabular}{lllll}
\hline $\mathbf{n}$ & COMPLICAÇÃO DA FERIDA & MI & SEXo & F.R. \\
\hline 1 & Hematoma local & MIE & Masc. & não \\
1 & Hematoma local & MIE & Fem. & não \\
1 & Deiscência parcial & MIE & Masc. & sim \\
1 & Celulite & MIE & Fem. & sim \\
1 & Hematoma + necrose de bordo & MID & Masc. & não \\
\hline
\end{tabular}

$\mathrm{MI}=$ membro inferior; $\mathrm{MIE}=$ membro inferior esquerdo; $\mathrm{MID}=$ membro inferior direito; Masc. = masculino; Fem. = feminino; $\mathrm{n}=$ número; F.R. = presença de fatores de risco.

reexploração operatória ou o prolongamento da internação hospitalar.

Com relação à dor na incisão operatória do membro inferior, nenhum paciente apresentou dor intensa e não foram necessários analgésicos parenterais para alívio da dor no membro inferior. A dor foi classificada em moderada em $4,3 \%$ dos pacientes, leve em $21,7 \%$ e ausente em $73,9 \%$.

\section{Avaliação da Qualidade da Veia}

\section{Avaliação Macroscópica}

Todas as veias examinadas durante o ato operatório foram consideradas adequadas. Foram observadas lesões macroscópicas em 2 casos. Uma lesão foi devido à secção parcial inadvertida com a tesoura durante a dissecção e a outra por avulsão de um ramo que não foi visualizado. Em ambos os casos foi possível a correção através da sutura da lesão com fio de polipropileno monofilamentar (Prolene (B) 7-0.

\section{Avaliação Histológica}

A preservação endotelial foi graduada como grau 1 em 86,9\% e grau 2 em 13\%. Portanto, não se observou dano celular em área maior que $10 \%$ da superfície endotelial. O desarranjo das fibras elásticas esteve presente em apenas 2 casos e não foi observada a rotura das fibras elásticas. A arquitetura tecidual da veia foi considerada normal em 95,7\%.

As Figuras 6 e 7 exemplificam a avaliação histológica de segmentos de veia safena magna, com as colorações de hematoxilina-eosina e Calleja.

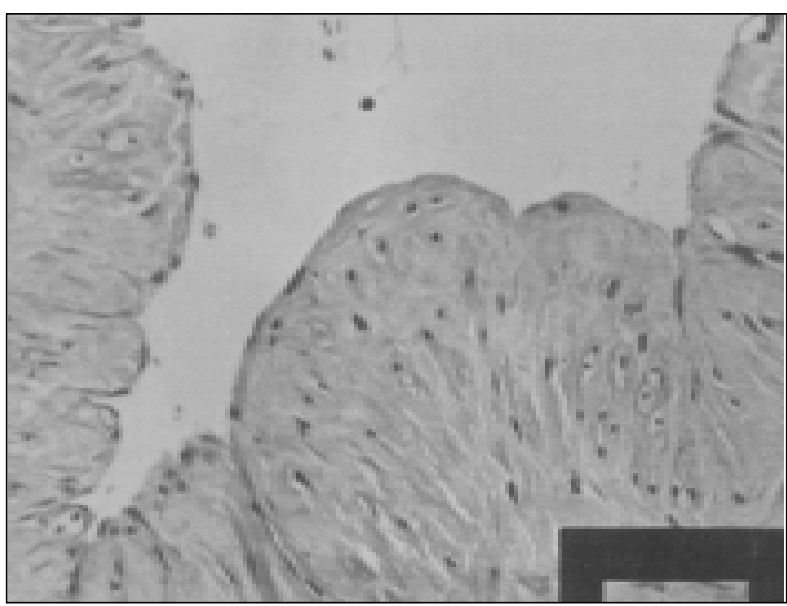

Fig. 6 - Fotografia do corte histológico de veia safena magna demonstrando a integridade endotelial-paciente 10 (Hematoxilinaeosina $-40 x$ )

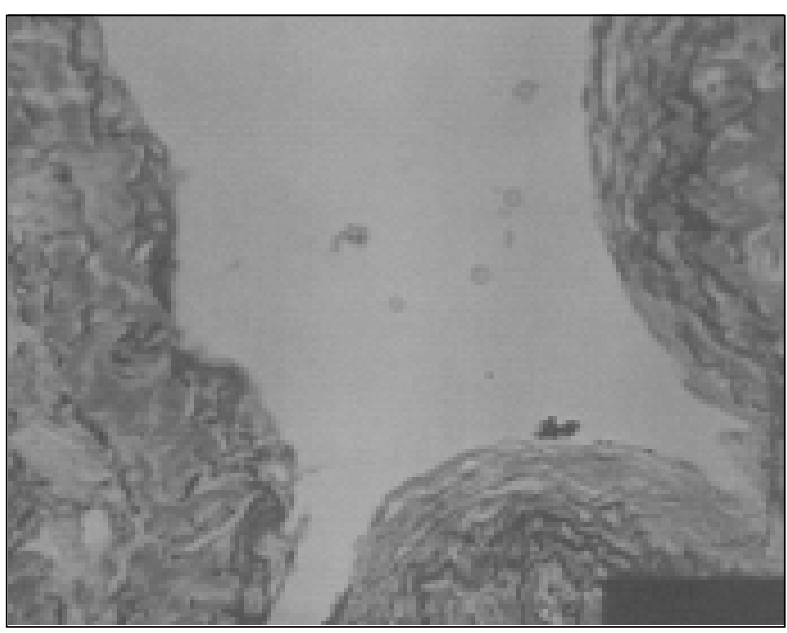

Fig. 7 - Fotografia do corte histológico de veia safena magna demonstrando a preservação da arquitetura tecidual e fibrose intimal grau 1 - paciente 10 (Calleja - 40x). 
Tyszka A L, Fucuda L S, Tormena E B, Campos A C L - Obtenção da veia safena magna através de acesso minimamente invasivo para revascularizações miocárdicas. Rev Bras Cir Cardiovasc 2001; 16(2): 105-13.

\section{COMENTÁRIOS}

Como o tratamento cirúrgico da isquemia miocárdica é uma das intervenções mais realizadas no mundo ocidental, técnicas que possam reduzir a freqüência de suas complicações resultarão em grande impacto na quantidade de pacientes beneficiados e nos sistemas de saúde. As técnicas minimamente invasivas visam a simplificar os procedimentos, reduzir o trauma operatório e são cosmeticamente mais atrativas aos pacientes (11). O presente estudo avaliou os resultados iniciais quanto à incisão operatória limitada e quanto à qualidade do enxerto venoso para a revascularização miocárdica.

A incidência de complicações com as incisões entre os pacientes do Grupo 2 ou grupo risco $(9,5 \%)$ e os pacientes do Grupo 1 ou grupo sem risco $(12 \%)$ não foi estatisticamente significativa, o que sugere que com as miniincisões estes fatores poderiam não ser considerados de risco. A ausência de complicações maiores em ambos os grupos reforça a utilização desta técnica especialmente para pacientes do sexo feminino, diabéticos, anêmicos, obesos ou com insuficiência arterial periférica, que seriam os mais beneficiados. Na casuística avaliada não se encontrou contra-indicações para o uso desta técnica.

O tamanho final das incisões determina menor quantidade de tecido que precisa ser seccionado e dissecado. A diminuição da solução de continuidade e da quantidade de tecido exposto ao meio externo facilita o processo cicatricial. A pele e o tecido celular subcutâneo que recobrem o túnel de onde a veia foi ressecada fornecem proteção para o tecido adiposo. A sutura da camada gordurosa subcutânea pode levar à necrose da gordura e liberação de potentes agentes inflamatórios (24). Ainda com maior importância a lesão cirúrgica dos canais linfáticos é, teoricamente, a principal causa da maioria das complicações da ferida ${ }^{(18)}$. A maior incisão pode romper maior número de linfáticos, principalmente na região inguinal onde a maioria destes vasos convergem para drenagem através do canal femoral. Além disso, durante o fechamento da ferida, as suturas podem comprometer ainda mais os canais linfáticos $(8,24)$.

Como anteriormente apontado por SLAUGHTER et al. ${ }^{(14)}$ esta modificação técnica é rápida e reprodutível. Em geral, o tempo para a obtenção do enxerto é inferior ao tempo para o preparo do paciente, para instalação da circulação extracorpórea e a dissecção da artéria torácica interna.

A incidência de problemas cicatriciais menores foi de $10,8 \%$, o que pode ser considerado inferior à maioria dos relatos na literatura com as incisões tradicionais. De acordo com a maneira como as complicações são definidas e relatadas, as suas incidências variam entre 23 a $43 \%(5,7-9)$. Excluídos os hematomas locais, que não fazem parte da maioria dos relatos, esta incidência diminui para $6,5 \%$ dos casos. Além disso, mesmo estas incisões resultaram em bom aspecto cicatricial, quando avaliadas após o décimo quinto dia de pós-operatório, não comprometendo a recuperação do paciente. Este tipo de incisão não apresentou complicações maiores, o que pode ocorrer com as tradicionais em 1 a $6 \%$ dos casos (6).

Enquanto o sucesso da operação e a recuperação do paciente são os principais objetivos de interesse ao cirurgião, a experiência pessoal do paciente e sua avaliação subjetiva do nível de conforto e resultado cosmético necessitam ser considerados ${ }^{(9)}$. Devido ao curto prazo de acompanhamento, o resultado estético final observado, embora bastante satisfatório, não pode ser considerado definitivo. O menor comprimento da incisão e o baixo índice de complicações, em teoria, se traduzem por resultado cosmético superior ao tradicional, com melhor nível de aceitação por parte dos pacientes. Por outro lado, ainda resta serem avaliadas alterações que poderiam ocorrer a longo prazo como a celulite recurrente e a retração cicatricial da incisão.

Estes acessos menos invasivos determinam menores queixas álgicas. A incisão através da região medial do joelho é local particular para desencadear dor, devido ao estiramento e tração na ferida e, portanto, a preservação desta região deve ser valorizada. A deambulação precoce é considerada importante fator na recuperação do paciente submetido à operação cardiovascular. Pacientes restritos ao leito estão mais sujeitos às complicações pulmonares e a falta de fisioterapia é uma das causas de internamentos mais prolongados.

A aterosclerose nos enxertos venosos é a principal causa de reoperação nos pacientes submetidos à revascularização miocárdica, maior do que a própria progressão da aterosclerose no leito coronariano nativo (25). A obstrução dos enxertos venosos, tanto precoce como tardia, tem a lesão endotelial como parte da patogênese. As regiões onde há perda das células endoteliais tem a sua atividade fibrinolítica diminuída e estão especialmente predispostas à agregação plaquetária e à trombose precoce. Fatores adicionais que podem lesar o endotélio, além do trauma cirúrgico, estão presentes durante a preparação do enxerto (26). A pressão de distensão, $0 \mathrm{pH}$ não fisiológico das soluções de preservação, a composição destes meios e a transitória perda de fluxo intraluminal com a isquemia resultante também são considera- 
Tyszka A L, Fucuda L S, Tormena E B, Campos A C L - Obtenção da veia safena magna através de acesso minimamente invasivo para revascularizações miocárdicas. Rev Bras Cir Cardiovasc 2001; 16(2): 105-13.

dos como potencialmente lesivos à parede da veia. O estudo microscópico da integridade endotelial após a dissecção demonstrou a preservação das células endoteliais. A graduação de preservação I e II são consideradas muito próximas da normalidade, com mínima perda de células endoteliais, que quando presentes $(<10 \%)$ pode ser considerado como artefato decorrente do preparo histológico dos fragmentos (22,23). A análise da interrelação do tecido conjuntivo com as fibras elásticas não mostrou desorganização ou rotura das fibras elásticas em $95,7 \%$ dos casos. A arquitetura histológica não foi alterada pela obtenção da veia através deste acesso.

\section{CONCLUSÕES}

O estudo permite concluir que a ressecção da veia safena magna, através de miniincisões de pele, é possível e permite obter comprimento adequado de enxerto venoso, disponível para a sua utilização como conduto nas revascularizações miocárdicas; a incidência das complicações pós-operatórias precoces com a miniincisão no membro inferior é baixa, mesmo em pacientes com fatores de risco e a qualidade do enxerto venoso, mediante a análise macroscópica e histológica, é considerada adequada para as revascularizações miocárdicas.

RBCCV 44205-536

Tyszka A L, Fucuda L S, Tormena E B, Campos A C L - Minimally invasive greater saphenous vein access as a conduit for coronary artery bypass surgery. Rev Bras Cir Cardiovasc 2001; 16(2): 105-13.

ABSTRACT: Objective: The aim of this study was to evaluate the possibility of excision of the greater saphenous vein through the minimal invasive technique, assessing its morphologic quality and the early complications in the leg wound.

Material and Methods: From July to November/1999, 46 patients received aortocoronary saphenous vein bypass graft. A miniincision was made, the vein was identified and gently dissected out with a special retractor, with a long and narrow blade. The samples of the vein were sent to histological analysis. Taking into accounts the presence or absence of the risk factors (female sex, anemia, obesity, peripheral vascular disease and diabetes mellitus) the patients were divided into two groups. The wounds were inspected, looking for majors and minor wound complications.

Results: The average number of incisions was 2.3 , the average length was $3.5 \mathrm{~cm}$, and the average of all incision length was $7.3 \mathrm{~cm}$. The average length of vein harvested was $34.1 \mathrm{~cm}$. The mean duration of the procedure was 28.7 minutes. Minor leg wound complications occurred in $5(10.8 \%)$ patients and the most common problem was local hematoma $(6.5 \%)$. There were no major complications. The macroscopically evaluation showed two tears but the correction was possible. Histological study showed that vascular integrity was maintained and there was no significant endothelial damage.

Conclusions: It was possible to harvesting satisfactory length of the vein through minimally invasive technique. This technique does not compromise the morphological quality of the vein graft. A low incidence of leg wound complications was observed, independently of the risk factors. These initial results suggest that this technique can be satisfactorily used for harvesting the greater saphenous vein. However, the long-term results still remain to be determined.

DESCRIPTORS: Myocardial revascularization. Cardiac surgical procedures, methods. Saphenous vein, surgery, methods. Surgical procedures minimally invasive. Saphenous vein, transplantation. 
Tyszka A L, Fucuda L S, Tormena E B, Campos A C L - Obtenção da veia safena magna através de acesso minimamente invasivo para revascularizações miocárdicas. Rev Bras Cir Cardiovasc 2001; 16(2): 105-13.

\section{REFERÊNCIAS BIBLIOGRÁFICAS}

1 Favaloro R G - Critical analysis of coronary artery bypass graft surgery: a 30-year journey. J Am Coll Cardiol 1998; 31(4 Suppl B): 1B-63B.

2 Loop F D, Lytle B W, Cosgrove D M et al. - Influence of the internal-mammary-artery graft on 10-year survival and other cardiac events. $N$ Engl $J$ Med 1986; 314: 1-6.

3 Lytle B W, Blackstone E H, Loop F D et al. - Two internal thoracic artery grafts are better than one. J Thorac Cardiovas Surg 1999; 117: 855-72.

4 Bruxton B, Acar C, Suma H et al. - Conduits. In: Buxton $\mathrm{B}$, Frazier $\mathrm{O} \mathrm{H}$, Westaby $\mathrm{S}$ - Ischemic heart disease surgical management. London: Mosby International Ltd, 1999; 139-77.

5 Lavee J, Schneiderman J, Yorav S, Shewach-Millet M, Adar R - Complications of saphenous vein harvesting following coronary artery bypass surgery. J Cardiovasc Surg 1989; 30: 989-91.

6 Lee K S \& Reinstein L - Lower limb amputation of the donor site extremety after coronary bypass graft surgery. Arch Phys Med Rehabil 1986; 67: 564-5.

7 DeLaria G A, Hunter J A, Goldin M D, Serry C, Javid $\mathrm{H}$, Najafi $\mathrm{H}$ - Leg wound complications associated with coronary revascularization. $J$ Thorac Cardiovasc Surg 1981; 81: 403-7.

8 Utley $\mathrm{J} R$, Thomason M E, Wallace $\mathrm{D} \mathrm{J}$ et al. Preoperative correlates of impaired wound healing after saphenous vein excision. $J$ Thorac Cardiovasc Surg 1989; 98: 147-9.

9 Wipke-Tevis D D \& Scotts N A - Effects of dressings on saphenous vein harvest incision pain, distress and cosmetic result. Prog Cardiovasc Nurs 1998; 13: 3-13.

10 Lutz C W, Schlensak C, Lutter G, Schollhorn J, Beyersdorf F - Minimal-invasive, video-assisted vein harvesting for cardiac and vascular surgical procedures. Eur J Cardiothorac Surg 1997; 12: 519-21.

11 Naiva J L \& Cosgrove 3rd DM - Minimally invasive mitral valve operations. Ann Thorac Surg 1996; 62: 1542-4.

12 Allen K B \& Shaar C J - Endoscopic saphenous vein harvesting. Ann Thorac Surg 1997; 64: 265-6.

13 Tevaearai H T - Dr. Cable's paper contributes important information. CTSNet Discussion Forums. jul. 1998. Discussion of: Cable D G, Dearani J A.
14 Pfeifer E A, Daly R C, Schaff H V - Minimally invasive saphenous vein harvesting: endothelial integrity and early clinical results. Ann Thorac Surg 1998; 66: 138-43.

15 Slaughter M S, Gerchar D C, Pappas P S - Modified minimally invasive technique for greater saphenous vein harvesting. Ann Thorac Surg 1998; 65: 571-2.

16 Dias R R, Jatene F B, Jatene A D - Dissecção minimamente invasiva da veia safena para obtenção de enxerto venoso na cirurgia de revascularização do miocárdio. Rev Bras Cir Cardiovasc 1997; 12: 244-7.

17 Allen K B, Griffith G L, Heimansohn D A et al. Endoscopic versus traditional saphenous vein harvesting: a prospective, randomized trial. Ann Thorac Surg 1998; 66: 26-32.

18 Hill S L - Relationship between ankle/arm blood pressure indices and healing of the harvest vein incision in coronary artery bypass patients. J Vasc Tech 1987; 11: $232-5$.

19 Horvath K D, Gray D, Benton L, Hill J, Swanstrom L L - Operative outcomes of minimally invasive saphenous vein harvest. Am J Surg 1998; 175: 391-5.

20 Folliguet T A, Le Bret E, Moneta A, Musumeci S, Laborde F - Endoscopic saphenous vein harvesting versus open technique: a prospective study. Eur $J$ Cardiothorac Surg 1998; 13: 662-6.

21 McGuire D B - The measurement of clinical pain. Nurs Res 1984; 33: 152-6.

22 Universidade Estadual de São Paulo. Faculdade de Medicina. Departamento de Patologia. Laboratório de Histologia (Colorações específicas) Botucatu - SP, 1996: 56.

23 Dries D, Mohammad S F, Woodward S C, Nelson R M - The influence of harvesting technique on endothelial preservation in saphenous veins. J Surg Res 1992; 52: $219-25$

24 Cable D G, Dearani J A, Pfeifer E A, Daly R C, Schaff $\mathrm{H} V$ - Minimally invasive saphenous vein harvesting: endothelial integrity and early clinical results. Ann Thorac Surg 1998; 66: 139-43.

25 El Gamel A, Dyde J, Perks J, Shaw R - Should we stitch the subcutaneous fat layer following saphenous vein excision for coronary revascularization? Eur $J$ Cardiothorac Surg 1994; 8: 162-4.

26 Loop F D, Lytle B W, Cosgrove D M et al. - Reoperation for coronary atherosclerosis: changing practice in 2509 consecutive patients. Ann Surg 1990; 212: 378-86. 